\title{
EFEITOS DA GINÁSTICA LABORAL NA DOR NAS COSTAS E NOS HÁBITOS POSTURAIS ADOTADOS NO AMBIENTE DE TRABALHO
}

\author{
DR. CLÁUDIA TARRAGÔ CANDOTTI \\ Doutora em Ciências do Movimento Humano pela Escola de Educação Física da \\ Universidade Federal do Rio Grande do Sul (ESEF/UFRGS) \\ Professora adjunta do Curso de Fisioterapia da Universidade Federal do \\ Rio Grande do Sul (Porto Alegre - Rio Grande do Sul - Brasil) \\ e-mail: claudia.candotti@ufrgs.br \\ GRAD. ROSEMERI STROSCHEIN \\ Graduada em Educação Física pela Universidade do Vale do Rio dos Sinos (UNISINOS) \\ (São Leopoldo - Rio Grande do Sul - Brasil) \\ e-mail: rose96@ibest.com.br \\ GRAD. MATIAS NOLL \\ Mestrando em Ciências do Movimento Humano pela Escola de Educação Física da \\ Universidade Federal do Rio Grande do Sul (ESEF/UFRGS) \\ Graduado em Educação Física pela Universidade do Vale do Rio dos Sinos (UNISINOS) \\ (Porto Alegre - Rio Grande do Sul - Brasil) \\ e-mail: matiasnoll@yahoo.com.br
}

\begin{abstract}
RESUMO
O objetivo deste estudo foi verificar o efeito da Ginástica Laboral (GL) sobre a dor nas costas e sobre os hábitos posturais de trabalhadores que ficam por longos períodos na posição sentada. Participaram 30 trabalhadores do setor administrativo, divididos em grupo controle $(n=15)$ e grupo experimental $(n=15)$, sendo este submetido a sessões de GL durante três meses. Ambos os grupos foram avaliados por um questionário de dor e postura, sendo as respostas codificadas, tabuladas e submetidas ao teste de Wilcoxon para verificar as diferenças entre pré e pós-experimento $(\alpha=0,05)$. Os resultados demonstraram que a $G L$ proporcionou diminuição da intensidade e frequência da dor referida aos trabalhadores do grupo experimental, e mudança do hábito postural durante o trabalho, melhorando a postura sentada.
\end{abstract}

PALAVRAS-CHAVE: Ginástica laboral; Iombalgia; postura, trabalhadores. 


\section{INTRODUÇÃO}

O mundo do trabalho vem sofrendo grandes mudanças devido às inovações tecnológicas e organizacionais, exigindo constantemente que os trabalhadores se adaptem às tecnologias e se atualizem perante um mercado cada vez mais competitivo (REIS et al., 2003; AUGUSTO et al., 2008). Pessoa, Cárdia e Santos (20 l0) relatam que estas mudanças e atualizações exigidas aos trabalhadores ocorrem em um ritmo muito elevado, geralmente maior que a própria capacidade humana pode suportar. Estes fatores, somado às extensas jornadas de trabalho, têm causado desconfortos musculares, afetando o bem-estar dos trabalhadores (FERNANDES; ASSUNÇÃO; CARVALHO, 2010 ; MENDES; LEITE, 2004; MUROFUSE; MARZIALE, 200I; CANDOTTI; NOLL; CRUZ, 20I0).

Vários estudos têm demonstrado que o estresse imposto pelas longas jornadas de trabalho, geralmente em más posturas e mobílias inadequadas, somados a movimentos repetitivos, em algumas profissões, resultam em alta prevalência de lombalgia e problemas posturais em trabalhadores (PASTRE et al., 2007; SOARES; ASSUNÇÃO; LIMA, 2006; MUROFUSE; MARZIALE, 200 I; FERNANDES; ASSUNÇÃO; CARVALHO, 20 I 0; PICOLOTO; SILVEIRA, 2008; ). Gurgueira, Alexandre e Corrêa Filho (2003) avaliaram trabalhadoras de enfermagem e constataram a existência de alta prevalência de sintomas osteomusculares nestes trabalhadores. Resultados idênticos demonstram a presença de lombalgia em garimpeiros (KHOURI et al., 2008), cirurgiões dentistas (SANTOS FILHO; BARRETO, 200 I), bancários (BRANDÃO; HORTA; TOMASI, 2005), manicures (CANDOTTI; NOLL; CRUZ, 20I0) e trabalhadores de indústrias de cerâmica (MELZER; IGUTI, 20I0).

Estes malefícios provenientes da atividade laboral resultam no afastamento de grande percentual de trabalhadores de suas atividades, sendo a principal causa de indenizações de auxílio doença e a terceira causa de aposentadoria por invalidez (PASTRE et al. 2007). Neste contexto, o impacto socioeconômico dos distúrbios osteomusculares ocupacionais vêm crescendo de forma preocupante, visto que, em todo o mundo, a prevalência de lombalgia e problemas osteomusculares vem atingindo proporções epidêmicas (MACIEL; FERNANDES; MEDEIROS, 2006).

Assim, na tentativa de contornar estes problemas, as empresas têm adotado a Ginástica Laboral (GL), como uma possibilidade da diminuição de afastamentos decorrentes de lesões por esforço repetitivo e doenças osteomusculares relacionadas ao trabalho, redução de atestados médicos, acidentes de trabalho e o aumento da produtividade (FIGUEIREDO; MONT'ALVÃO, 2005; MENDES; LEITE, 2004; MARTINS; DUARTE, 2000). 
Pressi e Candotti (2005) reforçam a ideia de que a inserção da GL na rotina das empresas pode apresentar resultados positivos, como o aumento da produtividade e disposição para o trabalho, melhoria da qualidade de vida e conscientização corporal. Entretanto, quanto ao efeito da GL na prevenção das lesões, lombalgia e distúrbios referentes a atividade laboral, a literatura ainda é controversa, necessitando de estudos mais aprofundados. Por exemplo, Augusto et al. (2008) relatam que a GL quando adotada de forma isolada, pode não ser eficaz para a prevenção. Deste modo justifica-se o presente estudo, devido a escassez de pesquisas experimentais, com boa descrição metodológica e procedimentos de coleta e análise válidos, que visem verificar os efeitos da GL em trabalhadores. Assim, este estudo teve como objetivo verificar se a Ginástica Laboral é uma ferramenta capaz de produzir efeitos positivos sobre a dor nas costas e sobre os hábitos posturais de trabalhadores que trabalham por longos períodos na posição sentada.

\section{MATERIAIS E MÉTODOS}

O estudo, do tipo experimental, com etapas de pré e pós-experimento, contou com a participação de 30 trabalhadores de ambos os gêneros, da cidade de Portão-RS, que trabalhavam no setor administrativo há no mínimo três anos, com carga horária diária de 8 a 10 horas. Os trabalhadores foram divididos em grupo experimental $(n=15)$ e controle $(n=15)$, não sendo demonstradas diferenças entre os grupos (Tabela I) nas questões antropométricas e quanto ao tempo de serviço. Todos os trabalhadores consentiram em participar voluntariamente do estudo a partir do Termo de Consentimento Livre e Esclarecido (TCLE).

Tabela I - Média e desvio-padrão da idade, massa corporal, estatura e tempo de serviço dos indivíduos dos grupos experimental e controle.

\begin{tabular}{lcccc}
\hline \multicolumn{1}{c}{ Grupos } & Idade (anos) & Massa corporal $(\mathrm{kg})$ & Estatura $(\mathrm{cm})$ & Serviço (anos) \\
\hline Experimental $(\mathrm{n}=15)$ & $32,1 \pm 10,9$ & $70,4 \pm 10,3$ & $163 \pm 0,06$ & $7,5 \pm 3,3$ \\
Controle $(\mathrm{n}=15)$ & $38 \pm 10,7$ & $74,0 \pm 10,8$ & $168,4 \pm 0,01$ & $6,9 \pm 3,2$ \\
Teste t & 0,145 & 0,351 & 0,103 & 0,619 \\
\hline
\end{tabular}

\section{PROCEDIMENTOS}

Este estudo foi desenvolvido em três fases distintas: (I) pré-experimento, realizado com o grupo experimental e controle, (2) experimento, caracterizado pelas sessões de GL realizada apenas com o grupo experimental e (3) pós-experimento, realizado com os grupos experimental e controle. 


\section{PROCEDIMENTOS DE AVALIAÇÃO}

Inicialmente foi solicitada e obtida e a autorização da empresa para o desenvolvimento da pesquisa, seguido pelo convite aos trabalhadores para que participassem da mesma. O estudo respeitou a Resolução 196/96 do Conselho Nacional de Saúde (BRASIL, 1996), de modo que foi garantido o sigilo de dados pessoais e confidenciais e os participantes do estudo não foram submetidos a nenhum grau de risco. Além disso, foram fornecidas, antes dos testes, informações detalhadas a respeito dos procedimentos do estudo. Todos os indivíduos participaram voluntariamente e assinaram um Termo de Consentimento declarando-se de acordo com sua participação na pesquisa.

Após esta etapa foi realizado um sorteio entre todos aqueles trabalhadores que aceitaram o convite para participar do estudo, com objetivo de formar, aleatoriamente, os grupos controle e experimental. Formados os grupos, todos os trabalhadores responderam um questionário de dor e postura, caracterizando assim a fase de pré-experimento. $\bigcirc$ mesmo procedimento foi novamente realizado com ambos os grupos após três meses caracterizando a fase de pósexperimento.

\section{INSTRUMENTO DE COLETA DE DADOS}

Foi utilizado um questionário, elaborado especificamente para este estudo, contendo sete questões fechadas sobre a temática dor e postura relacionada ao ambiente laboral (Anexo). Previamente a realização da coleta de dados, em um estudo piloto, foi avaliada a reprodutibilidade (fidedignidade) deste instrumento, com procedimento de teste e re-teste $(n=\mid 5)$ com quinze trabalhadores administrativos que não participaram dos grupos controle e experimental e intervalo de quinze dias entre o teste e o re-teste. Os dados do estudo piloto foram submetidos ao Teste de Correlação de Spearman e demonstraram que todas as questões apresentaram forte, muito forte ou perfeita correlação (CALLEGARIJAQUES, 2003) sendo todas significativas ( $p<0,00 \mathrm{I}$ ). Este resultado demonstrou a existência de reprodutibilidade do instrumento de avaliação. As respostas foram também submetidas ao teste de Wilcoxon, não apresentando diferenças entre teste e re-teste (Tabela 2). Deste modo conclui-se que o questionário de dor e postura demonstra-se como um instrumento adequado para a avaliação da dor e postura em trabalhadores. 
Tabela 2 - Resultados do procedimento de avaliação da reprodutibilidade do questionário de dor e postura

\begin{tabular}{cccc}
\hline & \multicolumn{2}{c}{ Teste de correlação de Spearman } & Teste de Wilcoxon \\
Questões & $r$ & $p$ & $p$ \\
\hline 1 & 1 & 0,000 & 1 \\
2 & 0,81 & 0,000 & 0,157 \\
3 & 0,83 & 0,000 & 0,18 \\
4 & 0,84 & 0,000 & 0,157 \\
$5 a$ & 0,83 & 0,000 & 0,056 \\
$5 b$ & 0,93 & 0,000 & 0,317 \\
$5 c$ & 1 & 0,000 & 1 \\
$5 d$ & 0,63 & 0,000 & 0,059 \\
5 e & 0,94 & 0,000 & 0,083 \\
6 & 1 & 0,000 & 1 \\
7 & 0,98 & 0,000 & 0,317 \\
\hline
\end{tabular}

\section{PROGRAMA DE GINÁSTICA LABORAL}

O programa de Ginástica Laboral envolveu sessões de GL, com duração de 15 minutos, realizada no horário de 7 h45min às $8 \mathrm{~h}$, com frequência de três vezes por semana, nas segundas, quartas e sextas-feiras. $\bigcirc$ programa foi desenvolvido em um período de três meses e as sessões consistiram em exercícios de alongamento (estáticos e dinâmicos), de mobilizações articulares, de exercícios de flexibilidade, resistência e relaxamento, com ênfase nos membros superiores e coluna cervical, visando preparar os trabalhadores para as atividades laborais que iriam iniciar. $\bigcirc$ programa de GL consistiu na fase de experimento deste estudo, sendo desenvolvido apenas com o grupo experimental.

\section{PROCEDIMENTOS DE ANÁLISE}

As respostas do questionário de dor e postura foram codificadas, tabuladas e analisadas no Software SPSS 15.0, sendo as variáveis nominais transformadas em variáveis numéricas. Excepcionalmente, a questão 5 do questionário teve um tratamento diferenciado, o qual inicialmente transformou as variáveis numéricas que refletiam a intensidade da dor em variáveis nominais. Exemplificando, a questão 5 foi classificada da seguinte forma: intensidade 0 (sem dor); I,2,3,4 (dor fraca); 
5 (dor moderada); 6,7,8,9 (dor forte) e 10 (dor muito forte). Estas categorias de dor foram então transformadas em novas cinco variáveis numéricas para que fosse possível realizar o tratamento estatístico.

Para a realização da análise estatística foram utilizados os procedimentos de estatística descritiva e inferencial. Para a validação do questionário foram utilizados os testes de Correlação de Spearman e de Wilcoxon. Para as variáveis: idade, massa corporal, estatura e tempo de serviço foram calculadas médias e desvios padrões e os dados submetidos ao teste $t$ para amostras independentes, para verificar as diferenças entre os grupos experimental e controle. Para verificar as diferenças entre os grupos experimental e controle, na fase de pré-experimento, as variáveis numéricas decodificadas dos questionários de dor e postura foram submetidas ao teste de Kruskal-Wallis. Para verificar as diferenças entre pré e pós-experimento, nos grupos experimental e controle, foi utilizado o teste de Wilcoxon. Além disso, os dados também foram organizados em tabelas de frequência. $\bigcirc$ nível de significância foi 0,05.

\section{RESULTADOS E DISCUSSÃO}

Os resultados da análise do questionário de dor e postura obtidos na fase de pré-experimento, demonstraram que não existe diferença significativa entre os grupos controle e experimental, tanto no que se refere a dor quanto a postura adotada durante a posição sentada (Tabela 3). Estes resultados sugerem que as diferenças que fossem ocorrer após o experimento seriam decorrentes do programa de GL. A única questão do questionário que apresentou diferença significativa entre os grupos nesta fase de pré-experimento foi quanto às intensidades da dor.

Os resultados do teste de Wilcoxon demonstraram que o grupo controle não apresentou diferenças na comparação entre pré e pós-experimento em nenhuma questão do questionário. Por outro lado, o grupo experimental apresentou diferença significativa entre pré e pós-experimento para as questões referentes à frequência da dor, interferência da dor nas AVDs, intensidade da dor e a postura no trabalho (Tabela 4).

Tabela 3 - Resultados do teste de Kruskal-Wallis na fase de pré-experimento,

comparando os grupos controle e experimental

\begin{tabular}{ll}
\hline Questão & Valor de $\mathrm{p}$ \\
\hline I. Você sente dor? & $\mathrm{I}$ \\
2. Em que horário esta dor se manifesta? & 0,277 \\
3. Qual a freqüência que você sente esta dor? & 0,416 \\
4. Esta dor interfere em suas atividades diárias? & 0,068 \\
5a. Circule nas escalas abaixo a intensidade da sua dor para braço: & 0,758 \\
5b. Circule na escala a intensidade da sua dor para punho e mão: & 0,007 \\
5c. Circule na escala a intensidade da sua dor para pescoço: & $0,020 *$ \\
5d. Circule na escala a intensidade da sua dor para as costas: & 0,000 \\
5e. Circule na escala a intensidade da sua dor para pernas: & 0,041 \\
\hline
\end{tabular}




\begin{tabular}{ll}
\hline Questão & Valor de $p$ \\
\hline 6. Você pratica atividade física regularmente, além da ginástica laboral? & 0,104 \\
7. Marque a figura que melhor representa sua postura durante o trabalho: & 0,285 \\
\hline
\end{tabular}

*Diferença significativa entre os grupos: $p<0,05$

Tabela 4 - Resultados do teste de Wilcoxon ( $p$ ) para os grupos controle e experimental, comparando as fases de pré e pós-experimento

\begin{tabular}{|c|c|c|}
\hline Questões & $\begin{array}{c}\text { Grupo } \\
\text { controle }\end{array}$ & $\begin{array}{c}\text { Grupo } \\
\text { experimental }\end{array}$ \\
\hline I. Você sente dor? & 1 & $\mathrm{I}$ \\
\hline 2. Em que horário esta dor se manifesta? & 0,181 & I \\
\hline 3. Qual a frequência que você sente esta dor? & । & $0,009 *$ \\
\hline 4. Esta dor interfere em suas atividades diárias? & 0,059 & $0,021 *$ \\
\hline 5a. Circule na escala a intensidade da sua dor para braço: & 0,059 & $0,007 *$ \\
\hline 5b. Circule na escala a intensidade da sua dor para punho e mão: & 0,105 & $0,031 *$ \\
\hline 5c. Circule na escala a intensidade da sua dor para pescoço: & I & $0,003 *$ \\
\hline 5d. Circule na escala a intensidade da sua dor para as costas: & 0,414 & $0,011 *$ \\
\hline 5e. Circule na escala a intensidade da sua dor para pernas: & 0,083 & $0,004 *$ \\
\hline 6. Você pratica atividade física regularmente, além da ginástica laboral? & 0,317 & 0,317 \\
\hline $\begin{array}{l}\text { 7. Marque a figura que melhor representa sua postura durante } \\
\text { o trabalho: }\end{array}$ & 0,181 & $0,015 *$ \\
\hline
\end{tabular}

*Diferença significativa entre pré e pós-experimento: $p<0,05$

A análise descritiva demonstrou que todos os trabalhadores de ambos os grupos referiram sentir dor na fase de pré-experimento, sendo que aproximadamente $20 \%$ dos trabalhadores, de ambos os grupos, apresentam dor forte e muito forte nos segmentos corporais avaliados (Tabela 5). Corroboram com o presente estudo, os resultados da pesquisa realizada com industriários onde foi demonstrado que houve uma alta prevalência de dor e que esta possui associação significativa com vários aspectos relacionados ao trabalho (MACIEL; FERNANDES; MEDEIROS, 2006). Do mesmo modo, o estudo realizado por Santos Filho e Barreto (200 I) em dentistas demonstrou que 58\% apresentaram queixa de dor músculo-esquelética em uma ou mais regiões corporais.

No presente estudo, na comparação entre pré e pós-experimento, observou-se também que para o grupo experimental houve diminuição das intensidades mais altas de dor em todos os segmentos (Tabela 5). Resultados opostos foram encontrados para o grupo controle, em que a intensidade da dor durante o trabalho permaneceu com poucas alterações em todos os segmentos corporais. Resultados idênticos são apresentados por Reis et al. (2003), onde verificou-se uma prevalência de lombalgia de $91 \%$, sendo diminuída em apenas 9\% com um programa de GL desenvolvido durante 6 meses. Independente disso, Soares, Assunção e Lima (2006) afirmam que a GL pode ser considerada uma 
medida eficaz para prevenir e combater os distúrbios físicos e emocionais na saúde do trabalhador, tais como estresse, lombalgias e doenças que o trabalho repetitivo e monótono pode acarretar.

Outro estudo (MARTINS; DUARTE, 2000) desenvolvido com trabalhadores participantes de sessões de GL de quinze minutos, três vezes por semana, durante quatro meses, demonstrou que houve melhora significativa do percentual de gordura, da pressão arterial e da flexibilidade. Porém não foram avaliados os efeitos da GL sobre a intensidade e frequência de lombalgia nos trabalhadores e, além disto, este estudo (MARTINS; DUARTE, 2000) contou apenas com grupo experimental, o que dificulta a inferência dos efeitos da GL.

A Tabela 6 apresenta os resultados referentes ao horário do dia em que a dor se manifesta. Pode-se observar que não houve alterações significativas nos horários em que os trabalhadores referem à dor para ambos os grupos. Na mesma tabela observa-se que os trabalhadores do grupo experimental referiram ter diminuído sua frequência de dor com as sessões de GL. Este resultado é relevante, pois demonstra que os trabalhadores que referiam ter dor diariamente passaram de $40 \%$ para apenas 6,7\%. Já o grupo controle não apresentou diferença entre as etapas de avaliação.

Os resultados da questão referente a interferência da dor nas AVDs demonstraram pouca diferença no grupo controle na comparação entre pré e pósexperimento. No entanto, com as sessões de GL, aproximadamente 30\% do grupo experimental descreveu que a dor na não interferia mais na execução das AVDs (Tabela 5), reforçando que a participação na GL influenciou positivamente na mudança de hábito nas posturas adotadas durante as AVDs (Tabela 4).

Considerando que o processo de envelhecimento e exposição continuada a rotinas profissionais e posturais inadequadas possuem forte impacto sobre a incidência das patologias posturais e dores referidas, entende-se que a diferença etária média existente entre os grupos controle e experimental (38 e 32 anos de idade, respectivamente) como uma limitação do estudo, que poderia ter influenciado os resultados do presente estudo.

Os resultados referentes à prática de atividade física regular, além da $\mathrm{GL}$, foram semelhantes entre pré e pós-experimento para ambos os grupos, demonstrando que a GL não influenciou na prática regular de atividade física dos participantes. Estes resultados são contrários aos achados de Dias et al. (2006), no qual verificou-se que 50\% dos participantes confirmam que a GL influenciou positivamente na adesão de atividade fisica regular, além de afirmar que a GL foi a principal influência para adesão desta prática. Assim, Maciel, Fernandes e Medeiros (2006) afirmam que caso a GL consiga conscientizar para a prática de atividade física regular, os trabalhadores estariam sujeitos a adaptações circulatórias e metabólicas, resultando em alterações benéficas na musculatura esquelética e tecidos conectivos. Essas alterações contribuiriam também para a diminuição do risco de surgimento de incapacidades e sintomas osteomusculares. 
Tabela 5 - Tabela de frequência da intensidade da dor durante o trabalho, por segmento do corpo, nas fases de pré e pós-experimento, para o grupo controle (A) e experimental (B).

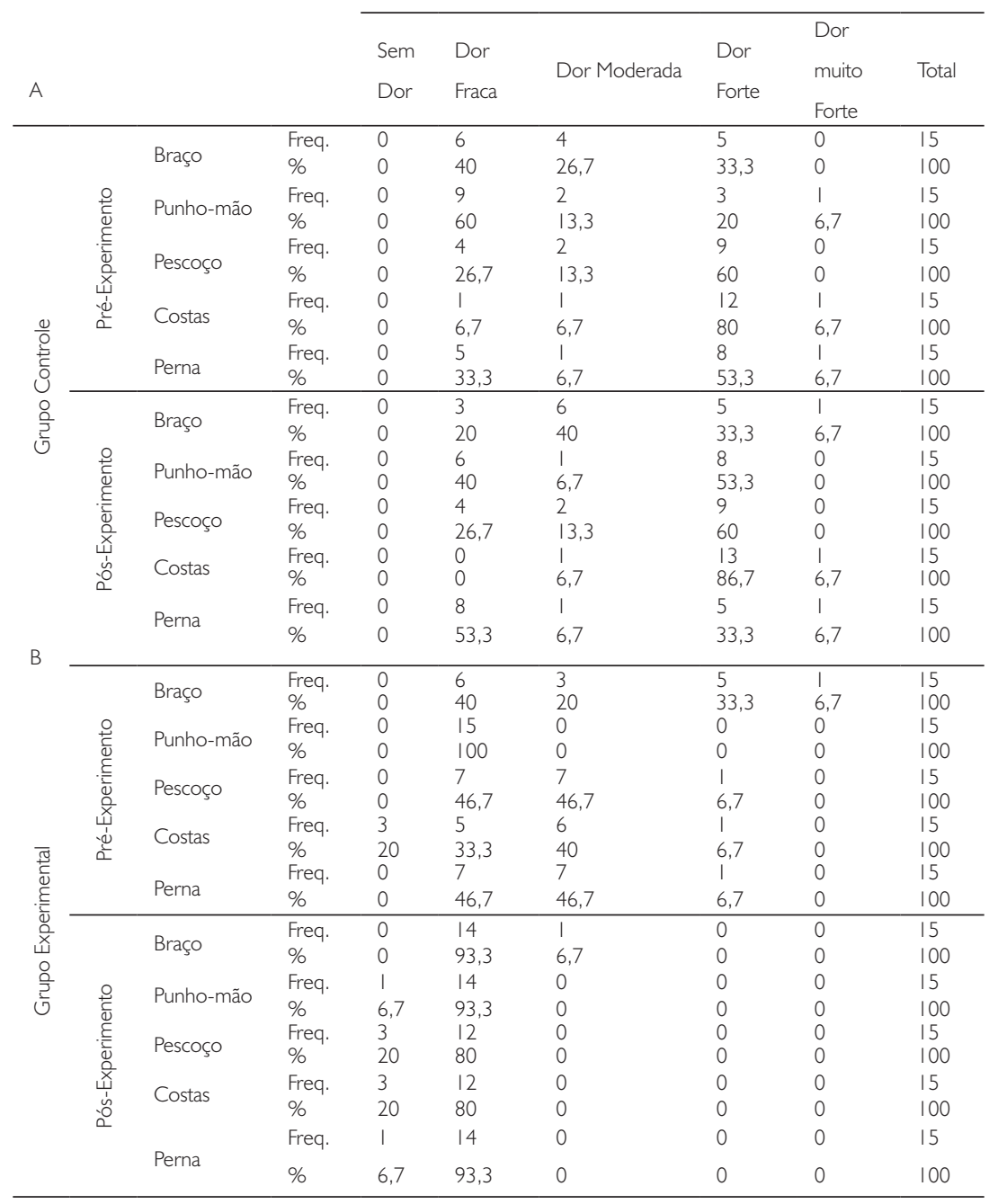

Os resultados da postura sentada adotada no trabalho (Tabela 4) demonstraram que no pré-experimento $100 \%(n=15)$ e $93,3 \%(n=14)$ apresentavam postura incorreta, para os grupos controle e experimental, respectivamente. Entretanto, após as sessões de GL, 53,3\% dos participantes do grupo experimental referiram sentar na postura correta. Já o grupo controle não apresentou mudanças entre as 
etapas de avaliação. Os achados encontrados no presente estudo demonstram a relevância da prática da GL na promoção de hábitos posturais corretos no ambiente de trabalho. Pressi e Candotti (2005) afirmam que a GL constitui-se como uma ação educativa que busca a conscientização para a promoção da saúde e qualidade de vida. Deste modo, especula-se que a lombalgia referida pelos indivíduos esteja associada a permanência por longos períodos em posturas incorretas, gerando desequilíbrios musculares, os quais remetem novamente a má postura, e um círculo vicioso é estabelecido (TOSCANO; EGYPTO, 200 I).

Tabela 6 - Tabela de frequência dos horários da manifestação de dor (A) e frequência semanal da dor (B), nas fases de pré e pós-experimento, para os grupos controle e experimental.

\begin{tabular}{|c|c|c|}
\hline \multirow[b]{3}{*}{ B } & \multicolumn{2}{|l|}{ Frequência (\%) } \\
\hline & Controle & Experimental \\
\hline & \multicolumn{2}{|l|}{ Pré-experimento } \\
\hline I a 2 vezes & $3(20)$ & $3(20)$ \\
\hline 3 a 5 vezes & $9(60)$ & $6(40)$ \\
\hline Todos os dias & $3(20)$ & $6(40)$ \\
\hline \multirow[t]{2}{*}{ Total } & $15(100)$ & $15(100)$ \\
\hline & \multicolumn{2}{|l|}{ Pós-experimento } \\
\hline I a 2 vezes & $3(20)$ & $9(60)$ \\
\hline 3 a 5 vezes & $9(60)$ & $5(33,3)$ \\
\hline Todos os dias & $3(20)$ & I $(6,7)$ \\
\hline \multirow[t]{3}{*}{ Total } & $15(100)$ & $15(100)$ \\
\hline & \multicolumn{2}{|l|}{ Frequência (\%) } \\
\hline & Controle & Experimental \\
\hline A & \multicolumn{2}{|l|}{ Pré-experimento } \\
\hline Antes do trabalho & $0(0)$ & $0(0)$ \\
\hline Durante trabalho & $5(33,3)$ & $8(53,3)$ \\
\hline Após trabalho & $10(66,7)$ & $7(46,7)$ \\
\hline \multirow[t]{2}{*}{ Total } & $15(100)$ & $15(100)$ \\
\hline & \multicolumn{2}{|l|}{ Pós-experimento } \\
\hline Antes do trabalho & I $(6,7)$ & $0(0)$ \\
\hline Durante trabalho & $6(40)$ & $8(53,3)$ \\
\hline Após trabalho & $8(53,3)$ & $7(46,7)$ \\
\hline Total & $15(100)$ & $15(100)$ \\
\hline
\end{tabular}


Andrade (200I) demonstrou que trabalhadores que permanecem sentados durante toda jornada de trabalho apresentam um estilo de vida sedentário e relevante fator de risco à saúde, particularmente às doenças cardiovasculares e metabólicas. Conforme Hallal et al. (20 I 0), apesar das evidências acumuladas sobre os benefícios da atividade física regular para a saúde, estudos indicam baixos níveis desse comportamento na população. Em contrapartida, Picoloto e Silveira (2008) demonstram que os programas de prevenção estão em plena expansão, inclusive, dentro das empresas, objetivando a melhora da qualidade de vida dos trabalhadores e a diminuição dos custos, principalmente com os afastamentos dos trabalhadores. Assim, embora o empresário lucre com a diminuição do absenteísmo e aumento da produtividade, é o trabalhador o maior beneficiado dos inúmeros benefícios desta atividade preventiva (MARTINS; DUARTE, 2000). De acordo com Zilli (2002), além da redução da dor e melhora da postura, a GL apresenta outros benefícios, entre eles, o aumento da integração entre os trabalhadores e melhora da vida social do trabalhador (diminuição de conflitos interpessoais, maior concentração e confiança e facilidade de trabalho em equipe). No entanto, no presente estudo estas questões não foram avaliadas, demonstrando a necessidade de novas pesquisas mais amplas a partir de estudos experimentais.

\section{CONCLUSÃO}

Os resultados demonstraram que a Ginástica Laboral foi eficaz na diminuição da intensidade e frequência da dor, e na correção dos hábitos posturais durante o trabalho, melhorando a postura sentada. Estes resultados sugerem que a Ginástica Laboral é uma ferramenta capaz de produzir efeitos positivos sobre a dor nas costas de trabalhadores que trabalham por longos períodos na posição sentada.

\section{Labor Gymnastic's effects on low back pain and postural habits adopted in the working environment}

ABSTRACT: The objective of this study aimed at verifying the Labor Gymnastic's (GL) effect on the low back pain and on postural habits of workers who stay for long periods in the sitting position. Thirty workers of both sex genders participated of the research; divided in two groups: control $(n=15)$ and experimental $(n=15)$, had GL sessions during three months. The instrument of evaluation consisted of a questionnaire about pain and posture, that the answers were codified and tabled as well as submitted to Wilcoxon test to verify differences between pre and post experiment in two groups. The results demonstrate that GL helped to diminish intensity and frequency of the referred pain in the workers from experimental group as well as changed their postural habit during the work.

KEYWORDS: Labor gymnastics; low back pain; posture; workers. 


\section{Efectos de Gimnasia en trabajo del dolor en la columna y habitos en postura adoptada en el contexto de trabajo}

RESUMEN: El objetivo de este estudio fue investigar el efecto de la Gimnasia Laboral (GL) en el dolor lumbar y en los hábitos posturales de los trabajadores. Participaron 30 trabajadores en el sector administrativo, divididos en grupo control $(n=15)$ y experimental $(n=15)$, que se sometieron a sesiones de GL durante tres meses. Ambos grupos fueron evaluados por un cuestionario sobre el dolor y la postura, con respuestas codificadas, tabulados y sometidos a la prueba de Wilcoxon para detectar diferencias entre el pre y post-experimento. Los resultados muestran que la GL ofrece a los trabajadores en el grupo experimental disminución en la intensidad y la frecuencia de dolor referido, y cambiar los hábitos posturales en el trabajo, mejorar la postura de sentado.

PALABRAS CLAVE: Gimnasia trabajo; dolor lumbar; postura; trabajadores.

\section{REFERÊNCIAS}

ANDRADE, A. Ocorrência e controle subjetivo do stress na percepção de bancários ativos e sedentários: a importância do sujeito na relação atividade física e saúde. Florianópolis, 2001 . 2v. 305 p. Tese (Doutorado em Engenharia de Produção) - Centro Tecnológico, Programa de Pós-Graduação em Engenharia de Produção Universidade Federal de Santa Catarina. Florianópolis, 2001.

AUGUSTO, V.G. et al. A look into Repetitive Strain InjuriMork-Related Musculoskeletal Disorders within physical therapists clinical context. Revista Brasileira de Fisioterapia, São Carlos, v. I2, n. I, p.49-58, jan./fev. 2008.

BRANDÃO, A.; HORTA, B.L.; TOMASI, E. Sintomas de distúrbios osteomusculares em bancários de Pelotas e região: prevalência e fatores associados. Revista Brasileira de Epidemiologia, São Paulo, v. 8, n.3, p. 295-305, set. 2005.

BRASIL. Ministério da Saúde. Conselho Nacional de Saúde. Normas de pesquisa envolvendo seres humanos. Res. CNS 196/96. Bioética, n. 4, p.15-25, 1996.

CALLEGARI-JAQUES, S. Bioestatística: princípios e aplicações. Porto Alegre: Artmed, 2003.

CANDOTTI, C.T.; NOLL, M.; CRUZ, M. Prevalência de dor lombar e os desequilíbrios musculares em manicures. Revista Arquivos em Movimento, Rio de Janeiro, v. 6, n. I , p. 125 40, jan./jun. 2010.

DIAS, G. et al. A contribuição de um programa de ginástica laboral para a aderência ao exercício físico fora da jornada de trabalho. Fitness \& Performance Journal, Rio de Janeiro, v. 5, n. 5, p. 325-32, set./out. 2006. 
FERNANDES, R. C. P.; ASSUNÇÃO, A. A.; CARVALHO, F. M. Repetitive tasks under time pressure: the musculoskeletal disorders and the industrial work. Revista Ciência e Saúde Coletiva, Rio de Janeiro, v. I5, n. 3, p. 931 -42, mai. 2010.

FIGUEIREDO, F;; MONT'ALVÃO, C. Ginástica Laboral e Ergonomia. Rio de Janeiro: Editora Sprint, 2005.

GURGUEIRA, G.; ALEXANDRE, N.; CORRÊA FILHO, H. R. Prevalência de sintomas músculo-esqueléticos em trabalhadoras de enfermagem. Revista Latino Americana de Enfermagem, Ribeirão Preto, v. I I, n. 5, p. 608-13, out. 2003.

HALLAL, P. C. et al. Evaluation of the Academia da Cidade program to promote physical activity in Recife, Pernambuco State, Brazil: perceptions of users and non-users. Revista Cadernos de Saúde Pública, Rio de Janeiro, v. 26, n. I, p. 70-78, abr. 2010.

KHOURI, M. et al. Prevalência de lombalgia em garimpeiros de Serra Pelada, Pará / Brasil. Acta Fisiátrica, São Paulo, v. I5, n. 2, p. 82-6, jun. 2008.

MACIEL, A. C. C.; FERNANDES, M. B.; MEDEIROS, L. S. Prevalence and factors associated with pain symptoms in professionals of the textile industry. Revista Brasileira de Epidemiologia, São Paulo, v. 9, n. I, p. 94-102, mar. 2006.

MARTINS, C.O.; DUARTE, M. F. S. Efeitos da ginástica laboral em servidores da Reitoria da UFSC. Revista Brasileira Ciência e Movimento, Brasília, v. 8, n. 4, p. 7-13, set. 2000.

MELZER, A.; IGUTI, A. Condições de trabalho e dor osteomuscular entre ceramistas brasileiros. Revista Cadernos de Saúde Pública, Rio de Janeiro, v. 6, n.3, p. 492-502, mar. 2010.

MENDES, R. A; LEITE, N. Ginástica Laboral: Princípios e Aplicações Práticas. Barueri: Editora Manole, 2004.

MUROFUSE, N. T.; MARZIALE, M. H.P. Mudanças no trabalho e na vida de bancários portadores de lesões por esforços repetitivos: LER. Revista Latino-Americana de Enfermagem, Ribeirão Preto, v. 9, n. 4, p. 19-25, jul. 2001.

PASTRE, E. et al. Work-related musculoskeletal complaints by women in a social rehabilitation center. Revista Cadernos de Saúde Pública, Rio de Janeiro, v 23, n. I I, p. 2605- I2, nov. 2007.

PESSOA, J. C.; CARDIA, M. C. G.; SANTOS, M. L. C. Analysis of the limitations, strategies and perspectives of the workers with RSIMRMD, participants of the PROFIT-LER Group: a case study. Revista Ciência e Saúde Coletiva, Rio de Janeiro, v. I5, n. 3, p. 82 I-30, maio 2010.

PICOLOTO, D.; SILVEIRA, E. Prevalência de sintomas osteomusculares e fatores associados em trabalhadores de uma indústria metalúrgica de Canoas-RS. Revista Ciência e Saúde Coletiva, Rio de Janeiro, v. 13, n. 2, p. 507-16, mar./abr. 2008.

PRESSI, A. M. S.; CANDOTTI, C.T. Ginástica Laboral. São Leopoldo: UNISINOS, 2005. 
SANTOS FILHO, S.; BARRETO, S. M. Atividade ocupacional e prevalência de dor osteomuscular em cirurgiões-dentistas de Belo Horizonte, Minas Gerais, Brasil. Revista Cadernos de Saúde Pública, Rio de Janeiro, v. I7, n. I, p. I81-93, jan./fev. 2001.

SOARES, R.; ASSUNÇÃO, A.; LIMA, F. Searching for elements at work that could explain the low attendance to a labor gymnastics program. Revista Brasileira de Saúde Ocupacional, São Paulo, v. 3 I , n. I I4, p. I49-60, jul./ago. 2006.

REIS, P. F. et al. O uso da flexibilidade no programa de ginástica laboral compensatória, na melhoria da lombalgia em trabalhadores que executam atividades sentados. In: CONGRESSO INTERNACIONAL DE EDUCAÇÃO FÍSICA, 18., 2003, Foz do Iguaçu. Anais..., Foz do Iguaçu, 2003.

TOSCANO, J.; EGYPTO, E. A influência do sedentarismo na prevalência de lombalgia. Revista Brasileira Medicina do Esporte, Niterói, v. 7, n. 4, p. 132-7, jul./ago. 2001.

ZILLI, C.M. Ginástica Laboral: Uma tarefa interdisciplinar com ação multiprofissional. São Paulo: LOVISE, 2002.

Recebido: 14 jun. 2010

Aprovado: 20 out. 2010

Endereço para correspondência:

Cláudia Tarragô Candotti

Laboratório de Pesquisa do Exercício (LAPEX) Sala 218

Escola de Educação Física (ESEF - UFRGS)

Rua Felizardo, 750 - Jardim Botânico - Porto Alegre - RS

CEP 90690-200

Fone: (5I) 3308-586 I 


\section{QUESTIONÁRIO}

NOME:

IDADE: MASSACORPORAL:

ESTATURA:

SETOR:

TEMPO NESTE CARGO:

I) Você sente dor? ( ) sim ( ) não :

2) Em que horário esta dor se manifesta?
a) antes do trabalho ( )
b) durante o trabalho ( )
c) após o trabalho ( )

3) Qual a freqüência que você sente esta dor?
a) I vez por semana ( )
b) 2 vezes por semana ( )
c) todos os dias ( )

4) Esta dor interfere em suas atividades diárias (lazer, afazeres domésticos, estudos, trabalho)

( ) $\operatorname{sim}$ ( ) não ( ) as vezes

5) Circule nas escalas abaixo a intensidade da sua dor, referente a cada segmento do corpo:

e) referente ao braço: $0-1-2-3-4-5-6-7-8-9-10$.

f) referente a punho/mão: $0-1-2-3-4-5-6-7-8-9-10$.

g) referente ao pescoço: $0-1-2-3-4-5-6-7-8-9-10$.

h) referente às costas: $0-1-2-3-4-5-6-7-8-9-10$.

i) referente às pernas: $0-1-2-3-4-5-6-7-8-9-10$.

6) Você pratica atividade física regularmente, além da ginástica laboral?

( ) $\operatorname{sim}($ ) não 
7) Marque a figura abaixo que melhor representa sua postura durante o trabalho:

(A)

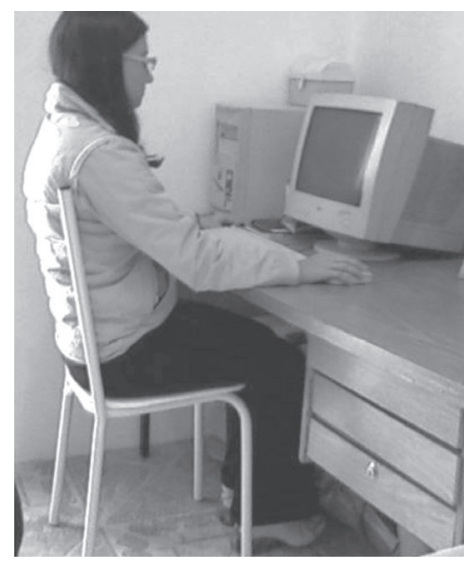

(C)

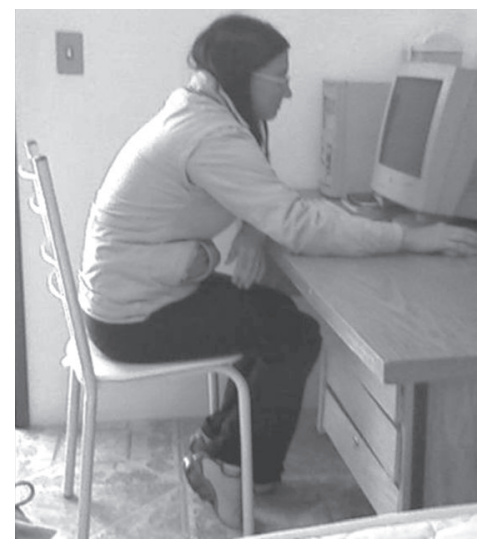

(B)

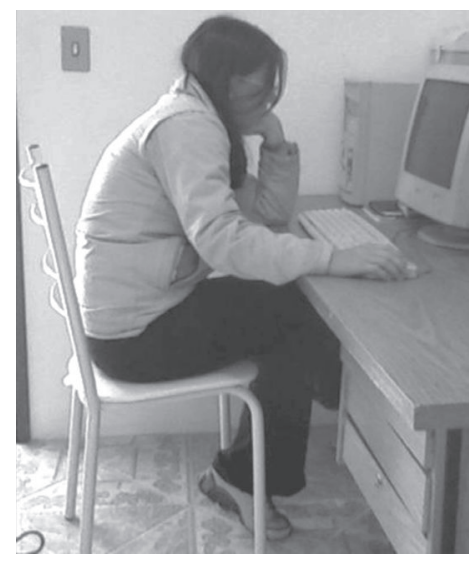

(D)

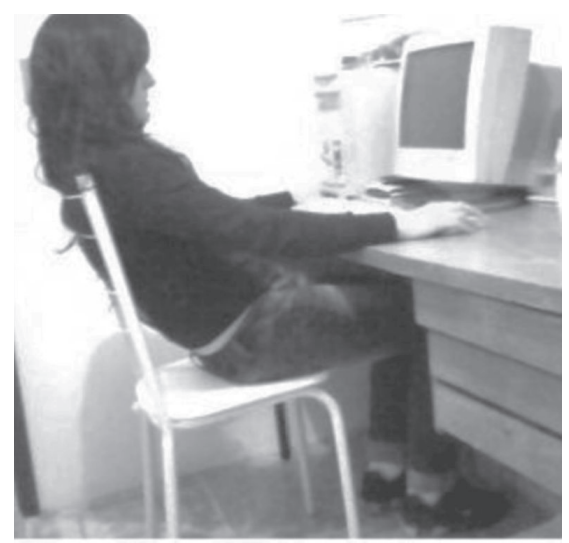

